\title{
Growing drift-cyclotron modes in the hot solar atmosphere
}

\author{
J. Vranjes ${ }^{1,2}$ and S. Poedts ${ }^{1}$ \\ 1 Centrum voor Plasma-Astrofysica, Celestijnenlaan 200 B, 3001 Leuven, Belgium \\ e-mail: [Jovo.Vranjes; Stefaan. Poedts] @wis . kuleuven . be \\ ${ }^{2}$ Faculté des Sciences Appliquées, avenue F.D. Roosevelt 50, 1050 Bruxelles, Belgium
}

Received 16 September 2007 / Accepted 17 January 2008

\section{ABSTRACT}

\begin{abstract}
Context. The ion cyclotron (IC) wave has been discussed in the literature in the context of the solar coronal heating. This is partly due to the necessity of explaining the observed preferential heating in the direction perpendicular to magnetic field lines. Observations have also shown the existence of filamentary density structures of various cross sections in the solar atmosphere. The presence of the related density gradients implies the possibility for the development of drift wave (DW) instability.

Aims. The frequencies of the two modes (IC and DW) are usually well separated, however, they can become close to each other for short inhomogeneity scale lengths of the equilibrium density. In this case, the drift wave can effectively couple to the ion cyclotron mode, and in the present work we want to demonstrate this coupling in the parameter domain relevant for the solar corona.

Methods. Well-known analytical results which follow from the kinetic theory are used and the dispersion equation, which describes coupled ion cyclotron and drift waves, is solved numerically.

Results. The numerical results obtained by using the values for the plasma density, magnetic field and temperature applicable to the solar corona clearly show the coupling and the instability (growing) of the two modes. The coupling happens at very short wavelengths, that are of the order of the ion gyro radius, and for characteristic scale lengths of the equilibrium density that are altitude dependent and may become of the order of only a few meters.

Conclusions. The demonstrated instability of the two coupled modes (driven by the equilibrium density gradient) is obtained by using a rigorous kinetic theory model and for realistic parameter values. The physical mechanism which is behind the coupling is simple and is expected to take place throughout the solar atmosphere and the solar wind which contain a variety of very elongated density structures of various sizes. The mode grows on account of the density gradient, it is essentially an ion mode, and its further dissipation should result in an increased ion heating.
\end{abstract}

Key words. Sun: oscillations - Sun: corona

\section{Introduction}

The ion cyclotron resonance and the ion cyclotron mode have been discussed in the context of problems related to the heating of the solar corona, e.g., Marsch et al. (1982), Cranmer et al. (1999), Cranmer (2000), Tu \& Marsch (2001), Markovskii (2001), Isenberg (2001), Hollweg \& Isenberg (2002). This is due to various reasons, such as the evidence obtained from in situ measurements in the solar wind and coronal holes of resonant ion cyclotron heating (Hollweg \& Isenberg 2002), and a preferential heating of coronal ions (with respect to electrons) which is most dominant in the direction perpendicular to the magnetic field lines. The damping of such IC waves is believed to be a good candidate for the consequent coronal heating and solar wind acceleration (Markovskii 2001). However, as in many wave-heating scenarios of the corona proposed in the past, there is usually the problem of the source for the required massive generation of such IC waves. In the literature, various effects have been proposed as sources for the IC mode, like currents (Forslund 1970; Toichi 1971), global resonant MHD modes (Markovskii 2001), etc. Yet, we note that these effects themselves need some source, so the problem is not solved but merely shifted to another problem. The multi-fluid description of the beam-driven IC wave excitation is presented in Mecheri \& Marsch (2007), where a tiny ion-beam population is assumed to originate from small scale reconnections. A rather large growth rate (of the order of $0.1 \Omega_{\mathrm{p}}$, where $\Omega_{\mathrm{p}}$ is the proton gyro-frequency) is obtained for relatively large values of the wave number, and for angles of propagation below $60^{\circ}$.

In one of the recent studies dealing with the generation of ion cyclotron waves in the solar atmosphere (Markovskii 2001) it is pointed out that the solar atmosphere contains density inhomogeneities of various scales with density gradients in the direction perpendicular to the magnetic field. Indeed, detailed studies performed in the past, which include direct observations, reveal the existence of ray-like structures that span from very large perpendicular cross sections, like in the case of polar plumes and streamers, down to very fine filamentary structures with a cross section of the order of a kilometer. More details on that issue are given in Woo \& Habbal (1997), Woo (1996), November \& Koutchmy (1996), Karovska \& Habbal (1991), and in references cited therein.

Fine filaments are visible even from ground-based observations like those during the eclipse in 1991 (November \& Koutchmy 1996), showing a slow radial enlargement of the structures (i.e., in the direction out of the Sun) which is consistent with a low-beta plasma. In situ measurements by Voyager 1 and Voyager 2 (Woo \& Habal 1997) show that the finest structures in the slow solar wind at around $9 R_{\odot}$ are about 3 times finer than those in the fast wind. Assuming a radial expansion, they conclude that the transverse sizes of these highly elongated structures at the Sun are below $1 \mathrm{~km}$. In Karovska \& Habbal (1991) an image restoration method is used to study the 
structures of the quiet Sun, with a spatial resolution of $5^{\prime \prime}$ and a temporal resolution of $5.5 \mathrm{~min}$. The contour maps presented in the work reveal the existence of numerous structures of various sizes.

The presence of density gradients in the direction perpendicular to the magnetic field lines implies the possibility of the existence of a drift wave, a mode with the unique feature of being unstable both in the kinetic and the fluid domain (hence the term universally unstable mode). Within kinetic theory, the drift mode is unstable even for a Maxwellian electron distribution. This is due to the inverse electron Landau damping effect. Within fluid theory, the mode is also unstable, due to the common effect of the electron collisions and the ion inertia, in the presence of a background density gradient. A comparison between the kinetic and the fluid instability (Goldston \& Rutherford 1995) reveals that the resistive one is dominant provided that the electron parallel mean-free path is smaller than the parallel wavelength. This is essentially the reason for the interest in the drift modes with very large parallel wavelengths and relatively short perpendicular wavelengths.

One particularly interesting feature of the drift wave is related to its intrinsic nonlinearity (Hasegawa \& Sato 1989). The nonlinear terms, which follow from the convective derivatives in the momentum equations, are comparable to the linear ones provided that $k_{\perp}^{2} \rho_{\mathrm{s}}^{2} e \phi /\left(\kappa T_{\mathrm{e}}\right)$ is of the same order as $\rho_{\mathrm{s}} / L_{n}$. Here, $\phi$ is the electrostatic wave potential, $\rho_{\mathrm{s}}=c_{\mathrm{s}} / \Omega_{\mathrm{i}}, \Omega_{\mathrm{i}}$ is the ion gyrofrequency, $c_{\mathrm{s}}^{2}=\kappa T_{\mathrm{e}} / m_{\mathrm{i}}, L_{n}$ is the scale length of the density gradient, and $\rho_{\mathrm{s}} / L_{n}$ is usually a small quantity. Hence, the mode becomes nonlinear even for very small perturbations provided that $k_{\perp} \rho_{\mathrm{S}}$ is not much larger than unity. Nevertheless, in the literature dealing with the waves and instabilities in solar plasma, the effects related to the density gradient and the consequent drift waves are usually disregarded.

In our recent publications, some aspects of the drift wave instability in the solar plasma have been discussed. The collisional coupling between the drift and kinetic Alfvén waves in the upper solar atmosphere (Vranjes \& Poedts 2006) reveals a strongly growing drift mode in the chromospheric plasma. The similar collisional instability of the mode, though with a much smaller increment, has been demonstrated also for the coronal plasma. In both cases, the kinetic Alfvén part of the mode is shown to be collisionally damped. In the presence of a plasma flow along the magnetic field lines the drift mode is subject to a reactive-type instability, provided that this background velocity has a gradient in the perpendicular direction. Such a problem has been discussed in our recent work (Saleem et al. 2007) dealing with solar spicules. The drift mode has been shown to be unstable for typical spicule characteristic lengths of the density and the shear flow gradients, i.e. in the range of a few hundred meters up to a few kilometers, yielding wave frequencies of the order of a few Herz.

One of the basic properties of the drift mode is the low frequency $|\partial / \partial t| \ll \Omega_{\mathrm{i}}$. In the case when this low-frequency limit is not well satisfied, there appears a coupling between the drift and ion-cyclotron modes. In the limit when $|\partial / \partial t| \sim \Omega_{\mathrm{i}}$, this coupling is very effective and well known (Ichimaru 1973). In the simplest case, it yields a coupled unstable drift-cyclotron mode with the instability driven by the plasma density gradient. The first demonstration of this instability was performed by Mikhailovskii \& Timofeev (1963).

To the best of our knowledge, the perpendicular density gradients, and the coupling of the drift and IC modes in the regime $\omega \sim \Omega_{\mathrm{i}}$ together with the consequent wave instability, have never been discussed in the context of the heating of the solar corona and the acceleration of the solar wind. In the present work we describe the basic instability of the mode to focus attention on the existence of such an instability that may be widespread and may contribute substantially to the problems discussed above. The term "fine structures", used in the text above for the structures observed in the solar corona still denotes spatial scales that are much larger than those used in our present study, in Sects. 2 and 3. In other words, there is no observational support for the existence of such tiny density inhomogeneities, and it is not expected in the near future. Yet, in view of the variety of density structures at larger and observable spatial scales, and having no obvious physical effect that would prevent inhomogeneities at scales that are even shorter than those observed so far, such micro scale plasma inhomogeneities cannot be excluded.

\section{Drift-cyclotron instability}

Following Ichimaru (1973), the plasma dielectric function in the case of a negligible parallel wave vector and for the frequency limit $\omega \sim \Omega_{\mathrm{i}}$, and $k_{\perp}=k_{y}=k$ within the kinetic theory for hot ions and electrons, is given by (in the local approximation)

$$
\begin{aligned}
\epsilon(k, 0, \omega)= & 1+\frac{k_{\mathrm{e}}^{2}}{k^{2}}\left[\frac{\omega_{* \mathrm{e}}}{\omega}+1-\Lambda_{0}\left(\beta_{\mathrm{e}}\right)\right] \\
& +\frac{1}{k^{2} \lambda_{\mathrm{D}}^{2}}\left\{1-\left(\omega-\omega_{*}\right)\left[\frac{\Lambda_{0}(\beta)}{\omega}+\frac{\Lambda_{1}(\beta)}{\omega-\Omega_{\mathrm{i}}}\right]\right\}
\end{aligned}
$$

Here,

$\beta_{\mathrm{e}}=k^{2} \frac{T_{\mathrm{e}}}{T_{\mathrm{i}}} \frac{m_{\mathrm{e}}}{m_{\mathrm{i}}} \rho_{L}^{2}<1, \quad \beta=k^{2} \rho_{L}^{2} \gg 1$,

$\omega_{*}=\omega_{* \mathrm{e}} T_{\mathrm{i}} / T_{\mathrm{e}}, \quad \omega_{* \mathrm{e}}=\frac{n_{0}^{\prime}}{n_{0}} \frac{T_{\mathrm{e}} k_{y}}{m_{\mathrm{e}} \Omega_{\mathrm{e}}}, \quad k_{\mathrm{e}}=\frac{\omega_{\mathrm{pe}}}{v_{\mathrm{Te}}}$,

$\Lambda_{n}(X) \equiv I_{n}(X) \exp (-X), \quad \rho_{L}=\frac{v_{\mathrm{Ti}}}{\Omega_{\mathrm{i}}}$,

$I_{n}$ denotes the modified Bessel function of the $n$th order, and the prime denotes the derivative in the direction perpendicular to both the wave-vector and the magnetic field. Using $\Lambda_{n}(\beta) \rightarrow(2 \pi \beta)^{-1 / 2} \exp \left(-n^{2} / 2 \beta\right)($ for $\beta \rightarrow \infty)$, we have $\Lambda_{0}(\beta) \simeq$ $1 /\left[(2 \pi)^{1 / 2} k \rho_{L}\right] \equiv \delta$. In view of the first expression in Eq. (2), we have $\Lambda_{0}\left(\beta_{\mathrm{e}}\right) \simeq 1-\beta_{\mathrm{e}}$, while from the second one we have $\delta \ll 1$. The dispersion equation then becomes (Ichimaru 1973)

$$
\begin{aligned}
& \left(1+k^{2} \lambda_{\mathrm{d}}^{2}-\delta\right) \omega^{2}-\left[\Omega_{\mathrm{i}}\left(1+k^{2} \lambda_{\mathrm{d}}^{2}\right)+\omega_{*}(1-\delta)\right] \omega+\omega_{*} \Omega_{\mathrm{i}}=0,(3) \\
& \lambda_{\mathrm{d}}^{2}=\rho_{L}^{2} \frac{m_{\mathrm{e}}}{m_{\mathrm{i}}}+\frac{1}{k_{\mathrm{e}}^{2}} \frac{T_{\mathrm{i}}}{T_{\mathrm{e}}}
\end{aligned}
$$

In the two limits $\left(\omega \ll \Omega_{\mathrm{i}}\right.$ and $\omega \sim \Omega_{\mathrm{i}}$ ), the two modes are the drift wave and the IC wave, respectively, $\omega_{1}=\omega_{*} /\left(1+k^{2} \lambda_{\mathrm{d}}^{2}\right)$ and $\omega_{2} \sim \Omega_{\mathrm{i}}\left[1+\delta /\left(1+k^{2} \lambda_{\mathrm{d}}^{2}\right)\right]$. The instability may appear at the point of eventual intersection of the two dispersion curves, and the instability condition reads:

$$
4 \omega_{*} \Omega_{\mathrm{i}}\left(1+k^{2} \lambda_{\mathrm{d}}^{2}-\delta\right)>\left[\Omega_{\mathrm{i}}\left(1+k^{2} \lambda_{\mathrm{d}}^{2}\right)+\omega_{*}(1-\delta)\right]^{2} .
$$

Below, we apply these expressions to the solar atmosphere in order to see if there is a window in the relevant parameter domain allowing for the instability. 


\section{Application to the solar atmosphere}

The necessary instability condition (4) can be satisfied for a chosen set of plasma parameters $n_{0}, T, B_{0}, L_{n}=\left(n_{0}^{\prime} / n_{0}\right)^{-1}$ and for a given wavelength. Because of the horizontal and vertical stratification, various values may be considered for the density, the temperature and the magnetic field. Yet, physically, in order to have an instability, the frequencies of the drift and IC modes must become close to each other, and this is most easily controlled by the density inhomogeneity scale-length $L_{n}$ and/or the wave-length. Here, $L_{n}$ is a local, spatially dependent parameter that determines the local properties of the drift-cyclotron mode. Assuming a cylindric elongated density structure with a radius $r_{0}$ and with a Gaussian radial density distribution

$n_{0}(r)=N_{0} \exp \left(-r^{2} / a^{2}\right)$,

where $N_{0}$ is the density at the axis of the cylinder, and $a$ determines the radial decrease of the density, we have $L_{n}(r)=$ $n_{0}(r) / n_{0}^{\prime}(r)=a^{2} /(2 r)$. Hence, we have a radially changing scalelength, which goes to infinity at the center and decreases towards the boundary. In the case of an e-folding decrease along $r$, we have $a=r_{0}, n_{0}\left(r_{0}\right) / N_{0}=37 \%$, and $L_{n} / r_{0}=1 / 2$. For such a density profile $L_{n}$ is minimum in the outer region of the plasma column. As a result, in the eigen-mode analysis of the drift wave in the cylindric geometry the amplitude and the increment of the drift mode are maximum in the same region Vranjes \& Poedts (2005); Bellan (2006).

Hence, Eq. (3) is solved numerically in terms of the wavelength and the density scale length, for parameter values applicable to the solar corona. As an example we take $B_{0}=10^{-3} \mathrm{~T}$, $n_{0}=10^{13} \mathrm{~m}^{-3}, T_{\mathrm{e}}=T_{\mathrm{i}}=10^{6} \mathrm{~K}$, that may be used to describe the physical properties of the plasma at the altitude of around one solar radius, and we have chosen a very short density inhomogeneity scale-length, $L_{n}=5 \mathrm{~m}$. In the case of the Gaussian profile discussed above and for the e-folding decrease, this yields the characteristic radius of the structure $r_{0} \simeq 10 \mathrm{~m}$. Such small values for $L_{n}$ are necessary to obtain high values for the drift wave frequency because it is proportional to $1 / L_{n}$. For these parameters, the plasma beta is 0.00035 and we have a proper electrostatic limit. The result is presented in Fig. 1. The instability develops in a narrow range of wavelengths around $1 \mathrm{~m}$. Note that the ion gyro radius for these parameters is equal to $\rho_{L}=0.94 \mathrm{~m}$. For larger wavelengths, the frequencies of the two modes become well separated and the instability vanishes. The maximum increment $\omega_{\mathrm{i}}$ is $\approx 5250 \mathrm{~Hz}$ at the real frequency $\omega_{\mathrm{r}}=102830 \mathrm{~Hz}$. This value of the increment is lower than the approximate theoretical value (Mikhailovskii \& Timofeev 1963) given by $\Omega_{\mathrm{i}}\left(m_{\mathrm{e}} / m_{\mathrm{i}}\right)^{1 / 4}$. The small values for $L_{n}$ imply a relatively short time for the existence of such structures, making them difficult to detect. As seen from Fig. 1, the frequencies of the corresponding modes are high, of the order of $10^{5} \mathrm{~Hz}$. On the other hand the ion collision frequency $v_{\mathrm{ii}}=4 n_{\mathrm{i} 0}\left(\pi / m_{\mathrm{i}}\right)^{1 / 2}\left[e_{\mathrm{i}}^{2} /\left(4 \pi \varepsilon_{0}\right)\right]^{2} L_{\mathrm{ii}} /\left[3\left(\kappa T_{\mathrm{i}}\right)^{3 / 2}\right]$ for the given parameters is about $10^{-2} \mathrm{~Hz}$. The perpendicular ion diffusion coefficient Chen (1988) is $D_{\perp} \approx \kappa T_{\mathrm{i}} v_{\mathrm{i}} /\left(m_{\mathrm{i}} \Omega_{\mathrm{i}}^{2}\right)=0.01 \mathrm{~m}^{2} / \mathrm{s}$. The diffusion velocity in the direction of the given density gradient is $D_{\perp} \nabla n / n=2 \mathrm{~mm} / \mathrm{s}$ only. So we have about 7 orders of magnitude difference for the two characteristic times, and this is enough time for the instability to develop before the equilibrium density structure disappears.

In Fig. 2, we fix the wave-length at $\lambda=0.95 \mathrm{~m}$, for the same parameter values as in Fig. 1, and calculate the frequency in terms of the density length scale $L_{n}$. The maximum increment $\omega_{\mathrm{i}}=5361 \mathrm{~Hz}$ is obtained at $L_{n}=5.3 \mathrm{~m}$, and the real part of

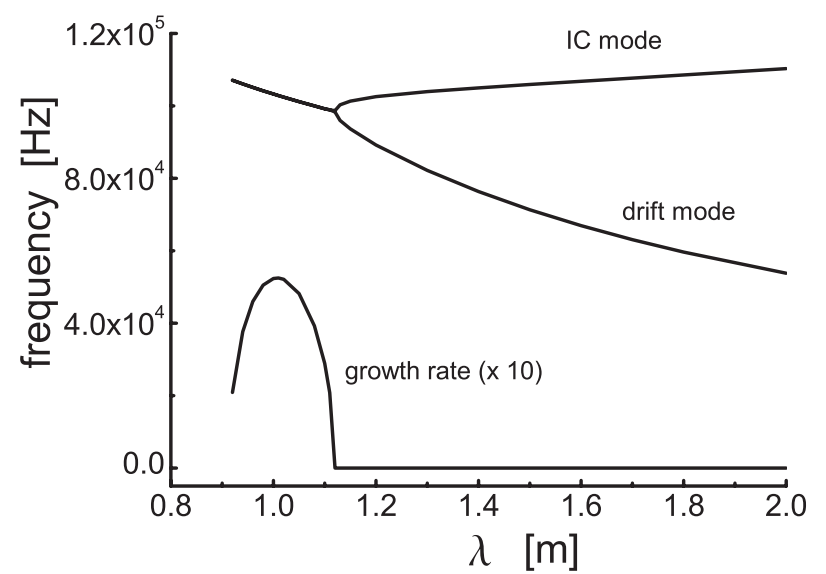

Fig. 1. The frequencies and the increment (multiplied by 10) of the coupled drift-cyclotron mode in terms of the wave-length.

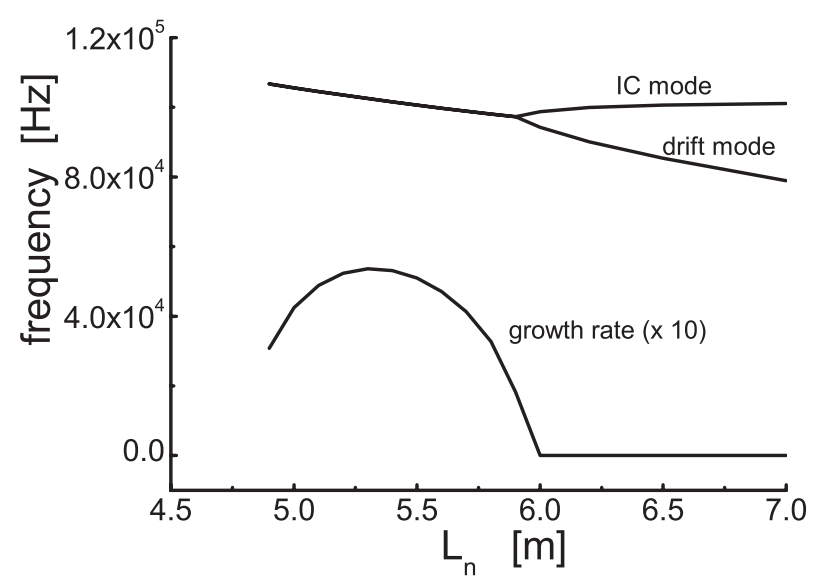

Fig. 2. The frequencies and the increment of the coupled drift-cyclotron mode in terms of the characteristic density gradient length. Here, the increment is multiplied by 10 .

the coupled mode frequency in that case is $\omega_{\mathrm{r}}=102504 \mathrm{~Hz}$. Compared to the recent results of Mecheri \& Marsch (2007), the present growth-rate driven by the density gradient appears to be smaller.

We note that in some studies, e.g., Coles \& Harmon (1989), the existence of a wave-number cutoff has been found at the ion inertial length $\lambda_{\mathrm{i}}=c / \omega_{\mathrm{pi}}$, preventing density perturbations for wavelengths shorter than $\lambda_{\mathrm{i}}$. However, this should not be confused with the density structures in our work. We are dealing with electrostatic perturbations at considerably shorter time and space scales that are presently difficult to detect. More importantly, there seems to be no definite consensus about the nature and the origin of the mentioned wave-length cutoff. Although it is a separate issue, in our view it seems very likely that the mentioned cutoff should be attributed to the problem of electromagnetic perturbations at spatial/time scales satisfying $(\omega / k)^{2} \ll c^{2}$, and for wavelengths below the ion inertial length. As it is known from plasma theory (Stamper \& Tidman 1973; Yu \& Stenflo 1985; Shukla et al. 2001; Vranjes et al. 2007a), in this case, the displacement current in the Ampère law $\nabla \times B=\mu_{0} \boldsymbol{j}+\mu_{0} \varepsilon_{0} \partial \boldsymbol{E} / \partial t$ can be omitted, and the ion perturbations become negligible. Further, setting Ampère's law (without the last term) in the electron continuity equation reveals that the electron density perturbations vanish. 


\section{Conclusions}

The instability discussed here implies very short scale lengths for the inhomogeneity of the equilibrium density and/or a very weak magnetic field. Only in such circumstances can the frequencies of the drift and IC modes become close to each other so that the two modes can effectively couple. Short density scale lengths are presently not directly observable in the solar atmosphere. However, we have learned that improvement of the resolution in observations of phenomena in the solar atmosphere tends to result in an increased variety and complexity of the density and/or magnetic field structures at short scales, showing very tiny filaments at scales below $1 \mathrm{~km}$. Therefore, one may expect a larger variety at even shorter scales. The present analysis clearly demonstrates the instability of perpendicularly propagating modes at frequencies in the range of the ion cyclotron frequency and at wavelengths of the order of the ion gyro radius. The tiny filaments are very elongated, they may extend to many solar radii, and the growth of the mode and the consequent dissipation and heating of ions may take place over large distances. The numbers used here are for the solar corona however, the large radial (from the Sun) length of the structures and the consequent decreasing of the magnetic field intensity implies larger density scale lengths at which the instability takes place. This can be easily shown by reducing the magnetic field to $10^{-4} \mathrm{~T}$ and the number density by one order of magnitude. As a result, the necessary density scale length $L_{n}$ for the unstable modes becomes of the order of 60 meters. Therefore the development of unstable growing modes may take place at large distances along the same density filaments that pervade the corona and spread within the solar wind. The presence of hotter ions in and around such filamentary structures should be interpreted as an indication and a signature of the instability.

There have been intensive searches for possible mechanisms for the excitation of such modes. Our analysis is based on one such mechanism that is well known, but not used in the context of the solar plasma, and it requires a step beyond the widely used MHD model. At higher densities or temperatures the plasma beta becomes higher so that electromagnetic effects should be included. However, it is known that eventual bending of the magnetic field lines implies an additional obstacle for electron motion along the magnetic lines, making the drift-type modes even more unstable Vranjes et al. (2007b). The parameter values that we use here are realistic and the instability that has been demonstrated for the given cases is thus physically very likely.

Acknowledgements. These results are obtained in the framework of the projects G.0304.07 (FWO-Vlaanderen), C 90205 (Prodex), GOA/2004/01 (K.U.Leuven), and the Interuniversity Attraction Poles Programme - Belgian State - Belgian Science Policy.

\section{References}

Bellan, P. M. 2006, Fundamentals of Plasma Physics (Cambridge: Cambridge Univ. Press), 257

Chen, F. F. 1988, Introduction to Plasma Physics and Controlled Fusion (New York: Plenum Press), 172

Coles, W. A., \& Harmon, J. K. 1989, ApJ, 337, 1023

Cranmer, S. R., Field, G. B., \& Kohl, J. L. 1999, ApJ, 518, 937

Cranmer, S. R. 2000, ApJ, 532, 1197

Forslund, D. W. 1970, J. Geophys. Res., 75, 17

Goldston, R. J., \& Rutherford, P. H. 1995, Introduction to Plasma Physics (Bristol and Philadelphia: Institute of Physics Pub.)

Hasegawa, A., \& Sato, T. 1989, Space Plasma Physics (Berlin Heidelberg: Springer-Verlag), 97

Hollweg, J. V., \& Isenberg, P. A. 2002, J. Geophys. Res., 107, 10.1029

Isenberg, P. A. 2001, Space Sci. Rev., 95, 119

Ichimaru, S. 1973, Basic Principles of Plasma Physics (The Benjamin/ Cummings Publish. Comp., Reading, Massachusetts), 186

Karovska, M., \& Habbal, S. R. 1991, ApJ, 371, 402

Markovskii, S. A. 2001, ApJ, 557, 337

Marsch, E., Goertz, C. K., \& Richter, K. 1982, J. Geophys. Res., 87, 5030

Mecheri, R., \& Marsch, E. 2007, A\&A, 474, 609

Mikhailovskii, A. B., \& Timofeev, A. V. 1963, Soviet Phys. JETP, 17, 626

November, L. J., \& Koutchmy, S. 1997, ApJ, 466, 512

Saleem, H., Vranjes, J., \& Poedts, S. 2007, A\&A, 471, 289

Shukla, P. K., Stenflo, L., \& Jovanovic, D. 2001, Phys. Scr., 64, 492

Stamper, J. A., \& Tidman, D. A. 1973, Phys. Fluids, 16, 2024

Toichi, T. 1971, Solar Phys., 18, 150

Tu, C. Y., \& Marsch, E. 2001, J. Geophys. Res., 106, 8233

Vranjes, J., \& Poedts, S. 2005, Phys. Plasmas, 12, 064501

Vranjes, J., \& Poedts, S. 2006, A\&A, 458, 635

Vranjes, J., Saleem, H., \& Poedts, S. 2007a, Phys. Plasmas, 14, 034504

Vranjes, J., Pandey, B. P., \& Poedts, S. 2007b, Phys. Plasmas, 14, 032106

Woo, R., \& Habbal, S. R. 1997, ApJ, 474, L139

Woo, R. 1996, Nature, 379, 321

Yu, M. Y., \& Stenflo, L. 1985, Phys. Fluids, 28, 3447 